\title{
Comments on "Continuity of care between hospital pharmacies and community pharmacies, and costs avoided: a pilot experience in times of COVID-19 in Spain"
}

\section{David Gómez Gómez}

Pharmacy Department, Marqués de Valdecilla University Hospital, Santander - Spain

Dear Editor,

The Pharmacy Department of the Marques de Valdecilla University Hospital (HUMV) would like to make a few clarifications about the results obtained by Zozaya et al (1) regarding the cost savings achieved by dispensing medications through community pharmacies during the COVID-19 pandemic.

It should be said that pharmaceutical care, which prior to the outbreak of the pandemic was typically carried out onsite on the hospital's premises, continued to be provided during the pandemic in a remote way, specifically by telephone, by hospital pharmacists, who gave patients the guidance and information they required on their treatment, facilitating pharmacotherapeutic follow-up and access to the prescribing physician and to their clinical record. The "informed deliveries" carried out by community pharmacists thus consisted of the delivery of the drug (2).

Furthermore, we would like to make two remarks regarding the social costs avoided. Firstly, the authors consider the lack of information on the patient's age or age group to be a limitation. In this regard, more than $50 \%$ of the patients whose treatment was provided by community pharmacists were not part of the working population (3). Furthermore, during the lockdown period only a limited number of people were able to go to work, basically those in essential services. Secondly, the Spanish Society of Hospital Pharmacists created a solidarity fund to finance drug deliveries which, if it had been made use of, would have avoided the increased costs faced by the hospital as a result of the exceptional circumstances that were taking place.

In addition, the authors state that the average number of drugs delivered through this initiative was more than

Received: March 20, 2021

Accepted: April 25, 2021

Published online: May 4, 2021

Corresponding author:

David Gómez Gómez

Pharmacy Department, Marqués de Valdecilla University Hospital

Avda. Valdecilla s/n 3

9003 Santander (Cantabria) - Spain

david.gomez@scsalud.es one-third of the drugs usually dispensed. However, these results are outdated (2015) and collected erroneously. In reality, in 2015, the number of daily outpatients was 168 (106 non-oncology and 62 oncology patients) and the number of medications delivered was 225 (163 non-oncology and 62 oncology patients). This discrepancy between patients and drugs is due to some patients being treated with more than one hospital drug. In 2020, the number of daily outpatients was 218 , so the average number of patients included in this initiative was a quarter of the outpatients at the HUMV.

Another consideration is the bias included in the survey carried out by the Chamber of Pharmacists of Cantabria. Only patients who agreed to be included in the initiative participated in this survey, but this represents a quarter of the patients seen at the HUMV. There was a high percentage of patients who refused to be included in this initiative and, therefore, their opinion was not reflected in the survey. The main reason for refusal was the lack of confidentiality, as patients did not feel comfortable with confidential information about their treatment being exchanged between hospital and community pharmacists.

Finally, extraordinary circumstances during the months of the study led to many adaptations of this process in order to keep providing patients with the pharmaceutical care they required. Consequently, these circumstances do not warrant the strong conclusions drawn by Zozaya et al (1).

\section{References}

1. Zozaya N, González-Domínguez A, Calvente N, et al. Continuity of care between hospital pharmacies and community pharmacies, and costs avoided: a pilot experience in times of COVID-19 in Spain. Glob Reg Health Technol Assess 2021;8:8-13. Online

2. Morillo-Verdugo $R$, Margusino-Framiñán $L$, Monte-Boquet $E$, et al. Spanish Society of Hospital Pharmacy Position Statement on Telepharmacy: Recommendations for its implementation and development. Farm Hosp. 2020;44(4):174-181. PubMed

3. Margusino-Framiñán L, Fernández-Llamazares $\mathrm{CM}$, NegroVega $E$, et al. ENOPEX: opinion and experience study on telepharmacy of patients in the COVID-19 pandemic in Spain. Previous results not published yet, sent to Am J Hosp Pharm October 30, 2020 for special number on Telemedicine. 